\title{
A novel early SKIP mode detection method for coarse grain quality in H.264/SVC
}

\author{
Hao Zhang ${ }^{*}$, Xiaoyu Zhu and Xuan He
}

\begin{abstract}
Various fast mode decision algorithms are proposed to reduce computational complexity for the H.264/scalable video coding standard. In this article, we focus on skip mode detection for coarse grain quality (CGS). We propose a fast skip mode detection approach by exploiting surrounding skip patterns, rate distortion (RD) cost and coded block pattern (CBP). Experimental results show that, with 0.589\% BDBR and $0.011 \mathrm{~dB}$ BDPSNR loss on average, the proposed method achieves considerable encoding time reduction compared with the reference software. Compared with the state-of-the-art early skip algorithm, the proposed method also achieves $9.98 \%$ time reduction on average.
\end{abstract}

\section{Introduction}

H.264 scalable video coding (SVC) can produce a single bitstream to adapt to various network conditions and hence it can be used to improve video quality over lossy networks [1]. Apart from its superior error resilience capabilities, the elimination of multipoint control unit (MCU) in video conference systems leads to great reduction of deployment cost and transmission delay [2]. Compared with H.264 single layer video coding, SVC requires coding of both base layer (BL) and enhancement layer (EL). To combat the additional bits required for multiple layers, SVC employs inter-layer prediction methods, including inter-layer intra prediction, residual prediction and motion prediction $[1,3]$. These additional layers and prediction modes greatly increase coding complexity. Therefore, efficient mode decision is necessary for real-time SVC applications.

In the literature, various fast mode decision (FMD) algorithms have been proposed to reduce H.264 computational complexity for single layer coding [4-8]. For H.264 SVC, there also exists considerable research study on FMD. Li et al. proposed a FMD algorithm in [9] where the RD costs of INTRA $4 \times 4$ and INTER $8 \times 8$ are checked to separate intra and inter modes. Moreover, reduction of candidate modes are performed by exploiting mode correlation between BL and EL. In [10], Park et al. proposed a FMD method with initial mode selection. In their method,

*Correspondence: hao@csu.edu.cn

School of Information Science and Engineering, Central South University, Changsha, Hunan, China
SKIP, BL_SKIP and INTRA $4 \times 4$ are first checked; then the mode with the highest expected RD cost (when skipped) is checked. A threshold is set for early termination. Kim et al. proposed a mode decision algorithm where a parameter is calculated from the weighted sum of all the modes from the neighboring MBs of BL and EL. Selected modes are then checked based on this parameter. In [11], an all-zeroblock (AZB) detection algorithm at EL is proposed. Based on the analysis of correlation of AZB occupance between $\mathrm{BL}$ and $\mathrm{EL}$, only some distributions are allowed to be AZB candidates for the EL. Furthermore, for a specific BL MB mode, priorities of $A Z B$ checking are given to increase the probability of AZB early detection. A new FMD algorithm is proposed in [12], where quantization parameter $(\mathrm{QP})$ values at both $\mathrm{BL}$ and $\mathrm{EL}$ and temporal layer indexes are used for mode decision. In [13], a novel FMD is proposed by exploiting the zero motion blocks and zero coefficient blocks to reduce the number of modes to be checked. A classification method is proposed in [14], where the mode in the first class (including SKIP and INTER16 $\times 16$ ) is first checked. Further mode checking would be performed depending on temporal layer indexes and whether all the resulted coefficients are zeros. Lin et al. attacked the FMD problem from another angle in [15]. They proposed a method to reduce the number of bi-directional predictions and thus greatly save computational time. Lee and Kim proposed a new FMD based on statistical hypothesis testing [16]. Mean and variance of pixels are used to decide early termination conditions. Zhao et al. proposed to use optimal stopping theory for early termination

\section{Springer}

(c) 2013 Zhang et al.; licensee Springer. This is an Open Access article distributed under the terms of the Creative Commons Attribution License (http://creativecommons.org/licenses/by/2.0), which permits unrestricted use, distribution, and reproduction in any medium, provided the original work is properly cited. 


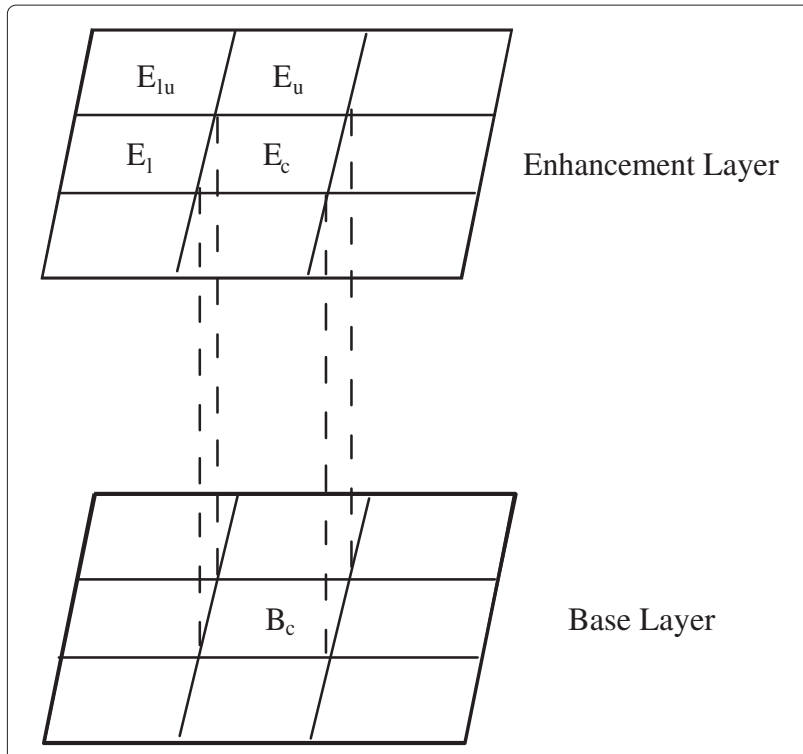

Figure 1 Relevant predictors for early skip mode detection.

decisions [17]. They classified all the candidate modes into modes without and with residual prediction (referred to as regular modes and residual modes). Optimal stopping theory is used for the regular modes. A reduced candidate set is used for residual modes based on the correlation between regular and residual modes. Zero block detection is also exploited to speed up the decision process of the residual modes. All the above FMD algorithms try to speed up the full mode decision process from different aspects. For example, mode correlation, expected $\mathrm{RD}$ cost, AZB detection are employed in [9-11], respectively. However, as an important part of FMD, skip mode decision is not studied as thoroughly as other parts of mode decision. Therefore, the scheme proposed in this article is complementary to other mode decision methods and would further increase performance gain. The only method that focuses on early skip is [18] proposed by Shen et al. for CGS (referred as Shen's algorithm). Their
Table 2 Percentage of SKIP and BL_SKIP MBs when Vs $=$ $(1,1,1)$ or $(1,1,0)$ or $(1,0,1)$ and Equations $(1),(2)$, and $(3)$ are satisfied

\begin{tabular}{ccc}
\hline $\left.\mathbf{Q P}_{\mathbf{B}}, \mathbf{Q P} \mathbf{E}\right)$ Sequence & $\mathbf{( 2 5 , \mathbf { 2 0 } )}$ & $\mathbf{( 3 5 , 3 0 )}$ \\
\hline Akiyo & $98.86 \%$ & $99.64 \%$ \\
Foreman & $90.60 \%$ & $97.44 \%$ \\
Silent & $98.44 \%$ & $99.12 \%$ \\
Mo_Da & $97.53 \%$ & $99.02 \%$ \\
Paris & $97.06 \%$ & $98.74 \%$ \\
Container & $98.11 \%$ & $99.31 \%$ \\
Coastguard & $94.32 \%$ & $96.27 \%$ \\
News & $97.66 \%$ & $99.14 \%$ \\
Average & $96.57 \%$ & $98.59 \%$ \\
\hline
\end{tabular}

approach decided a MB at EL to be a SKIP MB if the colocated $\mathrm{MB}$ at $\mathrm{BL}$, the top $\mathrm{MB}$ and the left $\mathrm{MB}$ are all encoded as SKIP mode. Although this approach achieves considerable speed boost with early SKIP mode detection, we find there is still much room for improvement in SKIP mode detection for CGS. In this article, we propose a fast early SKIP mode detection by considering other skip patterns, RD costs and CBP values. Shen's algorithm is the only method we are aware of that focuses on H.264 SVC early skip, therefore, we would compare our method with it throughout this article.

The rest of the article is organized as follows. Section 2 proposes a new SKIP detection algorithm based on SKIP mode distribution, RD cost differences and CBP values. Experimental results and conclusion are given in Sections 3 and 4.

\section{Proposed early SKIP algorithm}

\subsection{SKIP mode detection based on SKIP patterns}

H.264 SVC supports various inter-layer predictions as well as variable block size predictions. RD optimization and motion estimation are performed for each mode and consume a huge amount of computational power. SKIP

Table 1 Distribution for different $V s$ when $E_{c}$ is skipped

\begin{tabular}{|c|c|c|c|c|}
\hline \multirow[t]{2}{*}{$\left(Q P_{B}, Q P_{E}\right)$ Sequence } & \multicolumn{2}{|c|}{$(25,20)$} & \multicolumn{2}{|c|}{$(35,30)$} \\
\hline & $V s=(1,1,1)$ & $V s=(1,1,0),(1,0,1)$ & $V s=(1,1,1)$ & $V s=(1,1,0),(1,0,1)$ \\
\hline Akiyo & $88 \%$ & $8 \%$ & $92 \%$ & $6 \%$ \\
\hline Foreman & $42 \%$ & $34 \%$ & $56 \%$ & $29 \%$ \\
\hline Silent & $81 \%$ & $14 \%$ & $87 \%$ & $10 \%$ \\
\hline Mo_Da & $71 \%$ & $22 \%$ & $88 \%$ & $8 \%$ \\
\hline Paris & $68 \%$ & $23 \%$ & $74 \%$ & $20 \%$ \\
\hline Container & $79 \%$ & $15 \%$ & $90 \%$ & $8 \%$ \\
\hline Coastguard & $52 \%$ & $30 \%$ & $59 \%$ & $25 \%$ \\
\hline News & $78 \%$ & $16 \%$ & $84 \%$ & $12 \%$ \\
\hline Average & $70 \%$ & $20 \%$ & $79 \%$ & $15 \%$ \\
\hline
\end{tabular}


Table 3 Percentage of skipped MBs when Vs $=(1,1,1)$ and $\mathrm{CBP}=0$

\begin{tabular}{ccc}
\hline $\left.\mathbf{Q P}_{\mathbf{B}}, \mathbf{Q P} \mathbf{E}\right)$ Sequence & $\mathbf{( 2 5 , \mathbf { 2 0 } )}$ & $\mathbf{( 3 5 , 3 0 )}$ \\
\hline Akiyo & $98.33 \%$ & $99.48 \%$ \\
Foreman & $88.20 \%$ & $94.19 \%$ \\
Silent & $96.81 \%$ & $98.91 \%$ \\
Mo_Da & $90.79 \%$ & $98.46 \%$ \\
Paris & $96.57 \%$ & $98.26 \%$ \\
Container & $96.75 \%$ & $98.43 \%$ \\
Coastguard & $92.51 \%$ & $90.64 \%$ \\
News & $97.05 \%$ & $98.76 \%$ \\
Average & $94.62 \%$ & $97.14 \%$ \\
\hline
\end{tabular}

mode is prevalent at EL for low-bit rate SVC and requires little computation [18]. Simulations in [18] show that the average percentage for SKIP mode at EL is $59-71 \%$, while for low or medium motion sequences this percentage can be as high as 70\%. Based on this observation, Shen's algorithm performs early SKIP mode detection based on the co-located $\mathrm{MB}$ at $\mathrm{BL}$ and the neighboring left and top $\mathrm{MBs}$ at EL. For example, Figure 1 shows the relevant MBs for this early SKIP mode detection approach. As defined in [18], the current $\mathrm{MB}$ is denoted by $\mathrm{E}_{\mathrm{C}}$, the co-located $\mathrm{MB}$ at $\mathrm{BL}$ is denoted by $\mathrm{B}_{\mathrm{c}}$, the left, top and upper-left MBs at EL are denoted by $E_{l}, E_{u}, E_{l u}$, respectively. We define a 3-tuple $V s=\left(\operatorname{bskip}\left(B_{c}\right)\right.$, bskip $\left(E_{l}\right)$, bskip $\left.\left(E_{u}\right)\right)$, where bskip $\left(B_{c}\right)$ is a binary variable that is set to one if $B_{c}$ is a skipped MB and zero otherwise. Hence, Shen's algorithm is to determine $E_{c}$ to be a skipped $M B$ if $V_{s}=(1$, $1,1)$. When this happens, mode decision process is early terminated and encoding speed is greatly increased with small average PSNR loss. However, for some fast moving

Table 4 Encoder Configuration

\begin{tabular}{cc}
\hline Parameter & Setting \\
\hline JSVM version & 9.19 \\
No. of encoded frames & 32 \\
Frame rate & $30 \mathrm{fps}$ \\
Prediction structure & Hierarchial B \\
Scalability & CGS \\
Resolution & QCIF, CIF, HD720p \\
Group of pictures & 16 \\
No. of references & 1 \\
BL QP & $25-45$ \\
QP difference (DQP) & $2,5,10$ \\
ME search mode & Fast search \\
Search range & 16 \\
Entropy coding & CAVLC/BL, CABAC/EL
\end{tabular}

Table 5 Comparing the proposed early skip mode decision with JSVM

\begin{tabular}{ccccc}
\hline Sequence & DQP & BDPSNR & BDBR & ATS \\
\hline Akiyo & 2 & 0.024 & -0.246 & 56.52 \\
& 5 & -0.028 & 0.582 & 56.37 \\
Paris & 10 & -0.069 & 1.805 & 54.40 \\
& 2 & 0.009 & -0.009 & 36.49 \\
& 5 & -0.014 & 0.213 & 35.33 \\
Silent & 10 & -0.036 & 0.641 & 31.39 \\
& 2 & 0.014 & -0.154 & 48.43 \\
& 5 & -0.010 & 0.196 & 48.37 \\
Vidyo1 & 10 & -0.056 & 1.062 & 44.92 \\
& 2 & 0.103 & 0.003 & 51.85 \\
& 5 & -0.029 & 0.923 & 49.85 \\
Shields & 10 & -0.058 & 2.518 & 41.48 \\
& 2 & 0.001 & 0.029 & 16.05 \\
& 5 & -0.004 & 0.216 & 13.80 \\
& 10 & -0.025 & 1.455 & 7.60 \\
Average & 2 & 0.011 & -0.133 & 16.58 \\
& 5 & -0.001 & 0.135 & 13.37 \\
& 10 & -0.025 & 1.370 & 9.97 \\
& & -0.011 & 0.589 & 35.15 \\
& & & &
\end{tabular}

Table 6 Comparing the proposed early skip mode decision with Shen's algorithm

\begin{tabular}{ccccc}
\hline Sequence & DQP & BDPSNR & BDBR & ATS \\
\hline Akiyo & 2 & 0.036 & -0.400 & 15.74 \\
& 5 & 0.025 & -0.557 & 15.51 \\
Paris & 10 & 0.039 & -1.022 & 15.10 \\
& 2 & 0.084 & -1.354 & 15.47 \\
& 5 & 0.083 & -1.278 & 15.23 \\
Silent & 10 & 0.057 & -0.946 & 13.83 \\
& 2 & 0.016 & -0.206 & 14.94 \\
Vidyo1 & 5 & 0.018 & -0.306 & 14.70 \\
& 10 & 0.015 & -0.274 & 13.58 \\
& 2 & 0.061 & -0.893 & 9.14 \\
Shields & 5 & 0.060 & -1.652 & 9.41 \\
& 10 & 0.054 & -2.871 & 9.89 \\
& 2 & 0.007 & -0.250 & 2.52 \\
Average & 5 & 0.045 & -1.449 & 1.95 \\
\hline \multirow{2}{*}{ Mobcal } & 10 & 0.120 & -1.495 & -1.11 \\
& 2 & 0.010 & -0.303 & 5.52 \\
& 5 & 0.018 & -0.672 & 4.81 \\
& 10 & 0.064 & 0.105 & 3.43 \\
& & 0.045 & -0.879 & 9.98 \\
\hline & & & &
\end{tabular}


sequences, our analysis shows that this approach may result in non-negligible PSNR losses. Moreover, in the case $V s \neq(1,1,1)$, there are still quite some MBs at EL that can be skipped. Table 1 shows the statistical analysis with Joint Scalable Video Model (JSVM) for eight CIF sequences. We test 32 frames and GOP size and search range are set to 16 . In the table, $\mathrm{QP}_{\mathrm{B}}$ and $\mathrm{QP}_{\mathrm{E}}$ denote $\mathrm{QP}$ at $\mathrm{BL}$ and $\mathrm{EL}$, respectively. For each skipped $\mathrm{MB} \mathrm{E}_{\mathrm{c}}$ at EL, Vs values are collected. The table shows that, if $E_{c}$ is skipped, for the listed two QP settings, there is in

Table 7 Comparing the proposed algorithm with/without zero CBP detection

\begin{tabular}{|c|c|c|c|c|c|c|c|c|}
\hline \multirow[b]{2}{*}{ Sequence } & \multirow[b]{2}{*}{ QPB } & \multirow[b]{2}{*}{ QPE } & \multicolumn{3}{|c|}{ Without zero CBP detection } & \multicolumn{3}{|c|}{ With zero CBP detection } \\
\hline & & & Dbitrate & DPSNR & DTime & Dbitrate & DPSNR & DTime \\
\hline \multirow[t]{12}{*}{ Vidyo1 } & 40 & 38 & -1.63 & -0.05 & -50.69 & -1.66 & -0.05 & -51.98 \\
\hline & 35 & 33 & -0.85 & -0.03 & -52.26 & -0.85 & -0.03 & -53.47 \\
\hline & 30 & 28 & -0.18 & -0.02 & -51.62 & -0.31 & -0.02 & -52.89 \\
\hline & 25 & 23 & 0.59 & -0.05 & -48.56 & -0.03 & -0.05 & -49.08 \\
\hline & 40 & 35 & -1.21 & -0.06 & -49.76 & -1.14 & -0.06 & -50.98 \\
\hline & 35 & 30 & -0.68 & -0.05 & -51.58 & -0.68 & -0.04 & -53.09 \\
\hline & 30 & 25 & 0.73 & -0.05 & -51.24 & -0.02 & -0.04 & -51.91 \\
\hline & 25 & 20 & 1.68 & -0.04 & -44.96 & 0.70 & -0.04 & -43.40 \\
\hline & 40 & 30 & -0.55 & -0.07 & -49.32 & -0.56 & -0.07 & -50.37 \\
\hline & 35 & 25 & 0.96 & -0.06 & -50.91 & 0.13 & -0.06 & -51.80 \\
\hline & 30 & 20 & 2.13 & -0.07 & -45.90 & 0.82 & -0.06 & -44.76 \\
\hline & 25 & 15 & 1.01 & -0.01 & -20.26 & 0.69 & -0.01 & -19.01 \\
\hline \multirow[t]{12}{*}{ Shields } & 40 & 38 & -0.53 & -0.01 & -19.69 & -0.53 & -0.01 & -19.85 \\
\hline & 35 & 33 & -0.20 & -0.01 & -18.75 & -0.18 & -0.01 & -19.57 \\
\hline & 30 & 28 & 0.01 & -0.01 & -14.01 & -0.01 & -0.01 & -15.51 \\
\hline & 25 & 23 & 0.16 & 0.00 & -5.55 & 0.08 & 0.00 & -9.28 \\
\hline & 40 & 35 & -0.33 & -0.01 & -16.50 & -0.31 & -0.01 & -19.17 \\
\hline & 35 & 30 & -0.07 & -0.01 & -16.36 & -0.08 & -0.01 & -18.28 \\
\hline & 30 & 25 & 0.19 & -0.01 & -11.76 & 0.11 & -0.01 & -13.87 \\
\hline & 25 & 20 & 0.08 & -0.01 & -1.64 & 0.06 & -0.01 & -3.86 \\
\hline & 40 & 30 & -0.06 & -0.01 & -17.52 & -0.07 & -0.01 & -18.25 \\
\hline & 35 & 25 & 0.25 & 0.00 & -13.64 & 0.16 & -0.01 & -13.68 \\
\hline & 30 & 20 & 0.10 & -0.06 & -2.37 & 0.08 & -0.04 & -0.43 \\
\hline & 25 & 15 & 0.01 & 0.00 & 4.82 & 0.01 & 0.00 & 1.96 \\
\hline \multirow[t]{12}{*}{ Mobcal } & 40 & 38 & -0.84 & -0.01 & -24.06 & -0.82 & -0.01 & -26.12 \\
\hline & 35 & 33 & -0.26 & -0.01 & -20.33 & -0.24 & -0.01 & -21.77 \\
\hline & 30 & 28 & 0.02 & 0.00 & -11.24 & 0.01 & 0.00 & -13.19 \\
\hline & 25 & 23 & 0.08 & 0.00 & -3.11 & 0.04 & 0.00 & -5.23 \\
\hline & 40 & 35 & -0.41 & -0.01 & -21.61 & -0.38 & -0.01 & -22.50 \\
\hline & 35 & 30 & -0.11 & -0.01 & -17.96 & -0.11 & -0.01 & -18.91 \\
\hline & 30 & 25 & 0.21 & 0.00 & -9.22 & 0.14 & 0.00 & -9.82 \\
\hline & 25 & 20 & 0.03 & 0.00 & -0.44 & 0.02 & 0.00 & -2.30 \\
\hline & 40 & 30 & -0.04 & -0.01 & -19.00 & -0.04 & -0.01 & -21.85 \\
\hline & 35 & 25 & 0.23 & 0.00 & -11.44 & 0.15 & 0.00 & -14.05 \\
\hline & 30 & 20 & 0.04 & -0.07 & 0.22 & 0.03 & -0.05 & -3.42 \\
\hline & 25 & 15 & -0.01 & 0.00 & 2.22 & -0.01 & 0.00 & -0.59 \\
\hline Average & & & 0.02 & -0.02 & -23.22 & -0.13 & -0.02 & -24.51 \\
\hline
\end{tabular}


average 15 and $20 \%$ locations where only one of $E_{1}$ and $E_{u}$ is skipped (i.e., $V s=(1,1,0)$ or $\left.(1,0,1)\right)$. For some sequence such as Foreman with $\left(\mathrm{QP}_{\mathrm{B}}, \mathrm{QP} \mathrm{E}\right)=(25,20)$, this number is about $34 \%$ that is comparable to the case of Vs $=(1,1,1)$. If we can predict SKIP mode for these extra patterns with high precision, SKIP mode detection ratio at EL could be increased and so will encoding speed.

\subsection{SKIP mode detection based on RD cost comparison}

The second observation is based on the SKIP mode cost comparison. To facilitate description, we define SKIP RD cost as the RD cost resulting from only checking SKIP mode. $C(x)$ is used to represent the SKIP RD cost for any MB $x$. Please note that $C(x)$ is not the RD cost of the best mode (minimum RD cost) after the mode decision procedure is finished. It is equal to the minimum RD cost only when SKIP mode has been chosen as the best mode.

It can be observed that, for each skipped $M B E_{C}$ at $E L$, SKIP RD cost of $E_{c}$ is usually closer to the SKIP RD cost of the skipped neighboring $\mathrm{MB}$ than the non-skipped one. For instance, if the top $M B$ is skipped while the left $M B$ is not, the absolute difference $\left.\mid C\left(E_{c}\right)-C\left(E_{u}\right)\right) \mid$ is usually
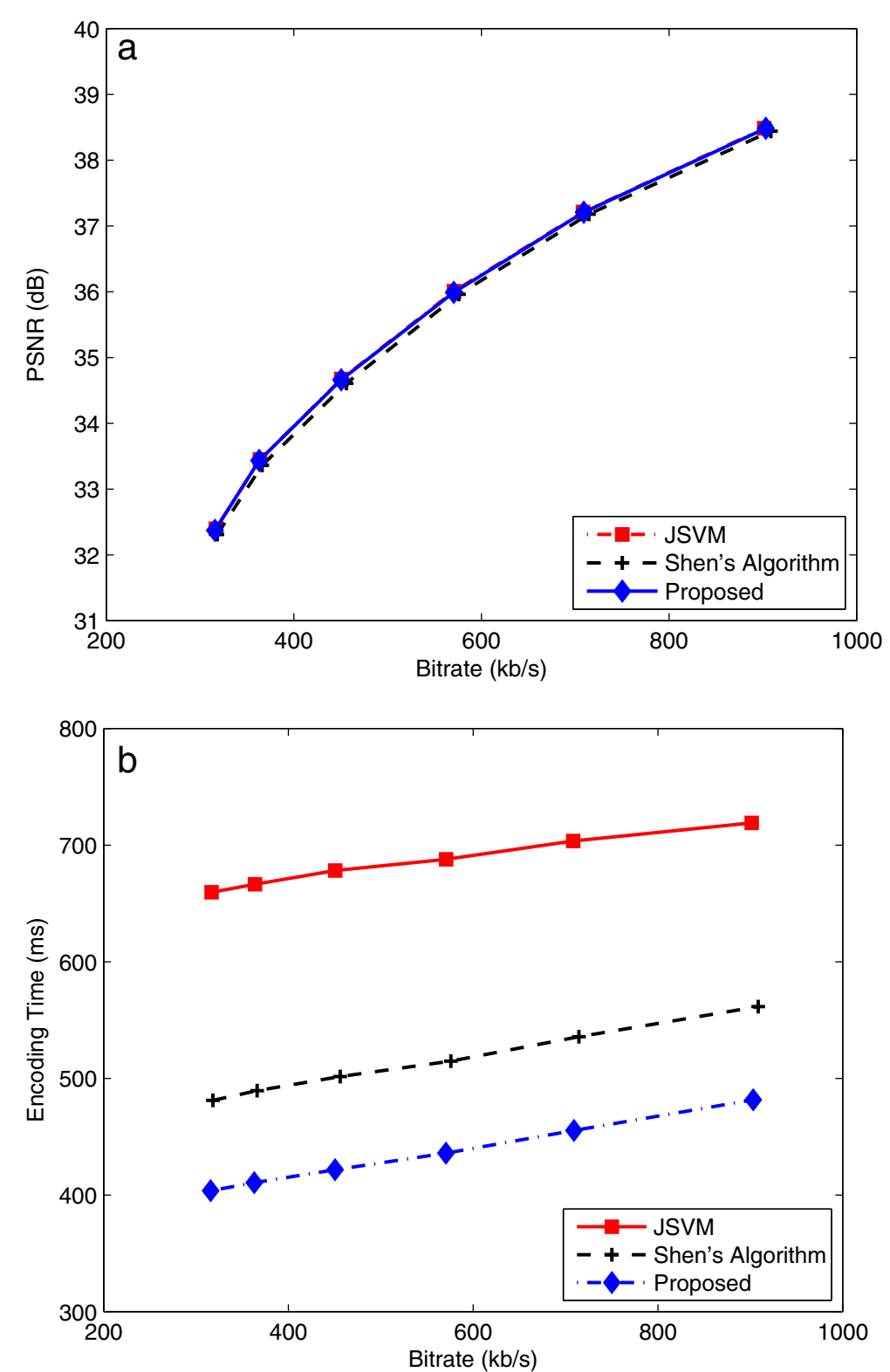

Figure 2 Comparison of RD performance (a) and encoding time per frame (b) for 'Paris' when DQP $=\mathbf{2}$. 
smaller than $\left.\mid C\left(E_{c}\right)-C\left(E_{l}\right)\right) \mid$. This observation could be conveyed by the following equations:

$$
\begin{aligned}
C\left(E_{c}\right) & \leq \frac{\alpha}{2}\left(C\left(E_{l}\right)+C\left(E_{u}\right)\right) \\
\left|C\left(E_{c}\right)-C\left(E_{l}\right)\right| & \left.\leq \alpha \mid C\left(E_{c}\right)-C\left(E_{u}\right)\right) \mid \\
\left|C\left(E_{c}\right)-C\left(E_{u}\right)\right| & \left.\leq \alpha \mid C\left(E_{c}\right)-C\left(E_{l}\right)\right) \mid
\end{aligned}
$$

In these equations, $\alpha$ is a configurable parameter and offers performance tradeoffs. Based on the analysis of many experimental results, we found $\alpha$ can be set to 1.5 to balance the encoding speed and video quality. Equation (1) is for the case of $V s=(1,1,1)$, i.e., all the $B_{c}, E_{l}, E_{u}$ are skipped. In this case, if $E_{c}$ is skipped, its SKIP RD cost is usually smaller than the average SKIP RD cost of the top and left MBs. Equations (2) and (3) correspond to Vs $=(1$, $1,0)$ and $V s=(1,1,0)$, respectively.

Second, when Vs $=(1,1,1)$ or $\mathrm{Vs}=(1,1,0)$ or $(1$, $0,1)$, aside from the SKIP mode, there is also a large number of MBs that are of BL_SKIP mode. Table 2 provides the distribution of SKIP and BL_SKIP mode when the cost conditions in Equations (1), (2), and (3) are
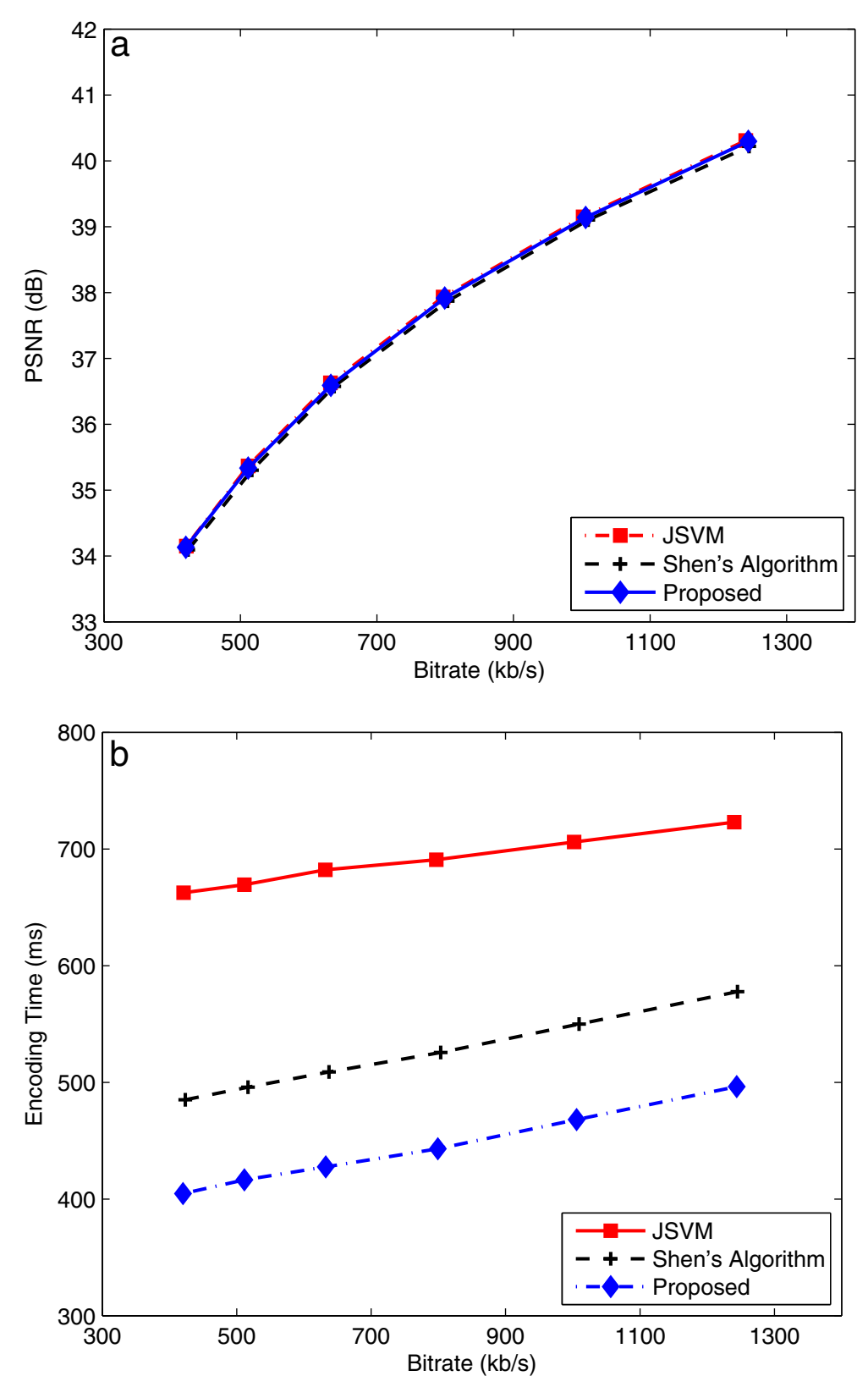

Figure 3 Comparison of RD performance (a) and encoding time per frame (b) for 'Paris' when DQP $=5$. 
satisfied. The table demonstrates that the average percentages are more than $95 \%$ when all the above conditions are satisfied. This implies the proposed early skip approach would predict the skipped MBs with high precision.

\subsection{Early SKIP by detecting zero CBP for mode direct $16 \times 16$}

Another way to decide skip mode before checking all other modes is by checking CBP after computing DIRECT16 $\times 16$ mode. We observed that SKIP mode would have high probability to be chosen if $\mathrm{Vs}=(1,1,1)$ and CBP for DIRECT16 $\times 16$ is zero. The validity of this observation can be verified through experimental results are shown in Table 3, where the percentages of skipped MB when CBP for DIRECT $16 \times 16$ is zero are displayed. It can be seen from the table that, for most cases, the percentages are more than $90 \%$, and the average values are $94.62 \%$ and $97.14 \%$ for $\left(\mathrm{QP}_{\mathrm{B}}, \mathrm{QP}_{\mathrm{E}}\right)=(25,20)$ and $\left(\mathrm{QP}_{\mathrm{B}}, \mathrm{QP}_{\mathrm{E}}\right)=(35,30)$, respectively. We also integrate this observation into our algorithm.

The overall skip mode detection method is based on all the observations described in Sections 2.1, 2.2, and 2.3. The whole procedure is described as follows. To make
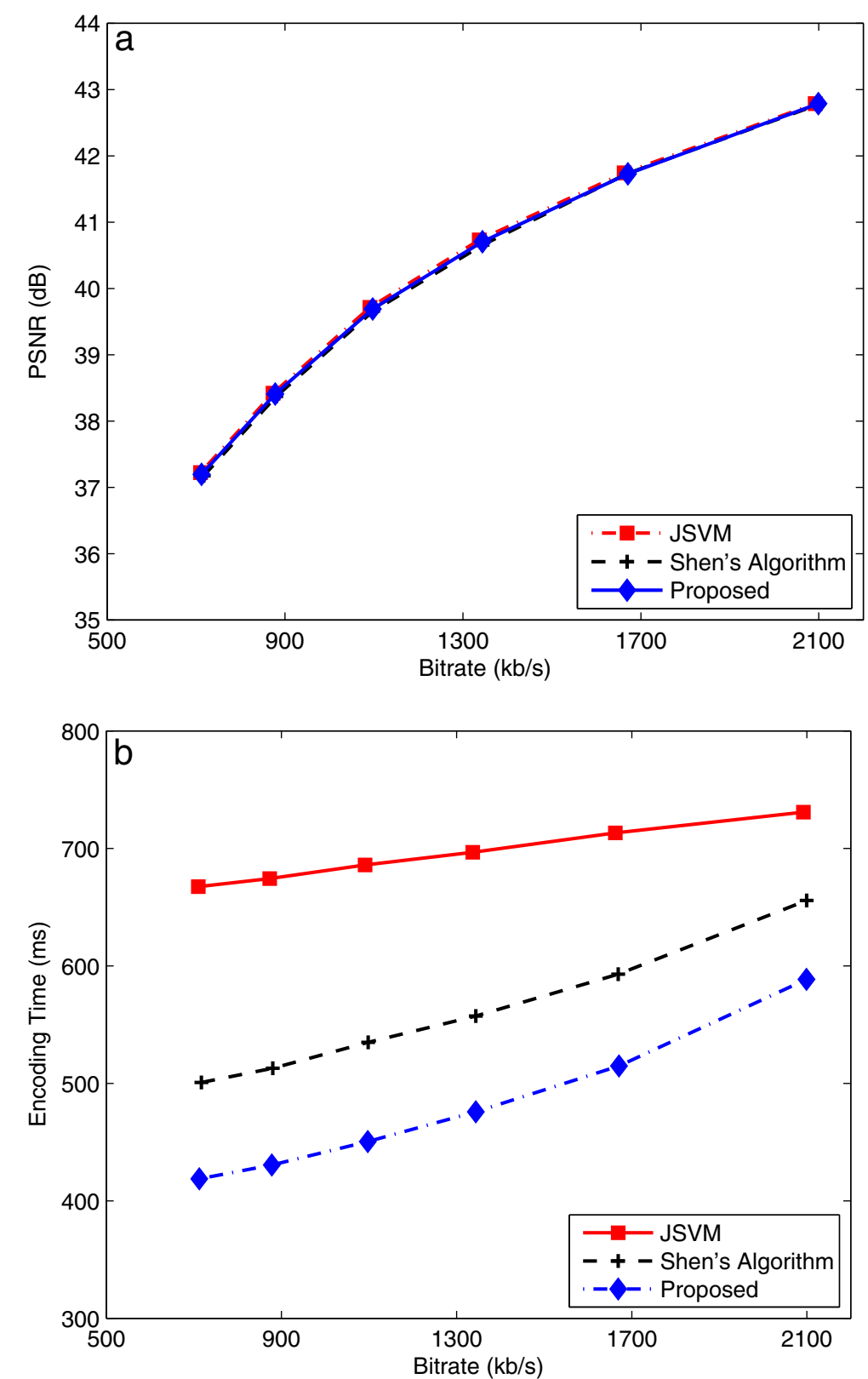

Figure 4 Comparison of RD performance (a) and encoding time per frame (b) for 'Paris' when DQP $=10$. 
the steps clearer, procedures for P slices and B slices are separated.

Procedure for P slices:

Step 1) For each MB, if $\mathrm{Vs}=(1,1,1)$ or $(1,1,0)$ or $(1,0$, 1), goto STEP 2; otherwise, goto to STEP 3.

Step 2) If $\mathrm{Vs}=(1,1,1)$ and Equation (1) is not satisfied, or Equations (2) and (3) are not satisfied for $\mathrm{Vs}=(1,1,0)$ and $\mathrm{Vs}=(1,0,1)$, respectively, goto STEP 3; otherwise, check BL_SKIP mode and goto STEP 4.
Step 3) Conduct the normal exhaustive mode decision for the rest of modes and determine the best mode.

Step 4) Go to STEP 1 to process the next MB.

Procedure for B slices:

Step 1) For each $M B$, if $V s=(1,1,1)$ or $(1,1,0)$ or $(1,0,1)$, goto STEP 2; otherwise, goto to STEP 5 .

Step 2) If $\mathrm{Vs}=(1,1,1)$, goto STEP 3 ; otherwise, goto STEP 4.
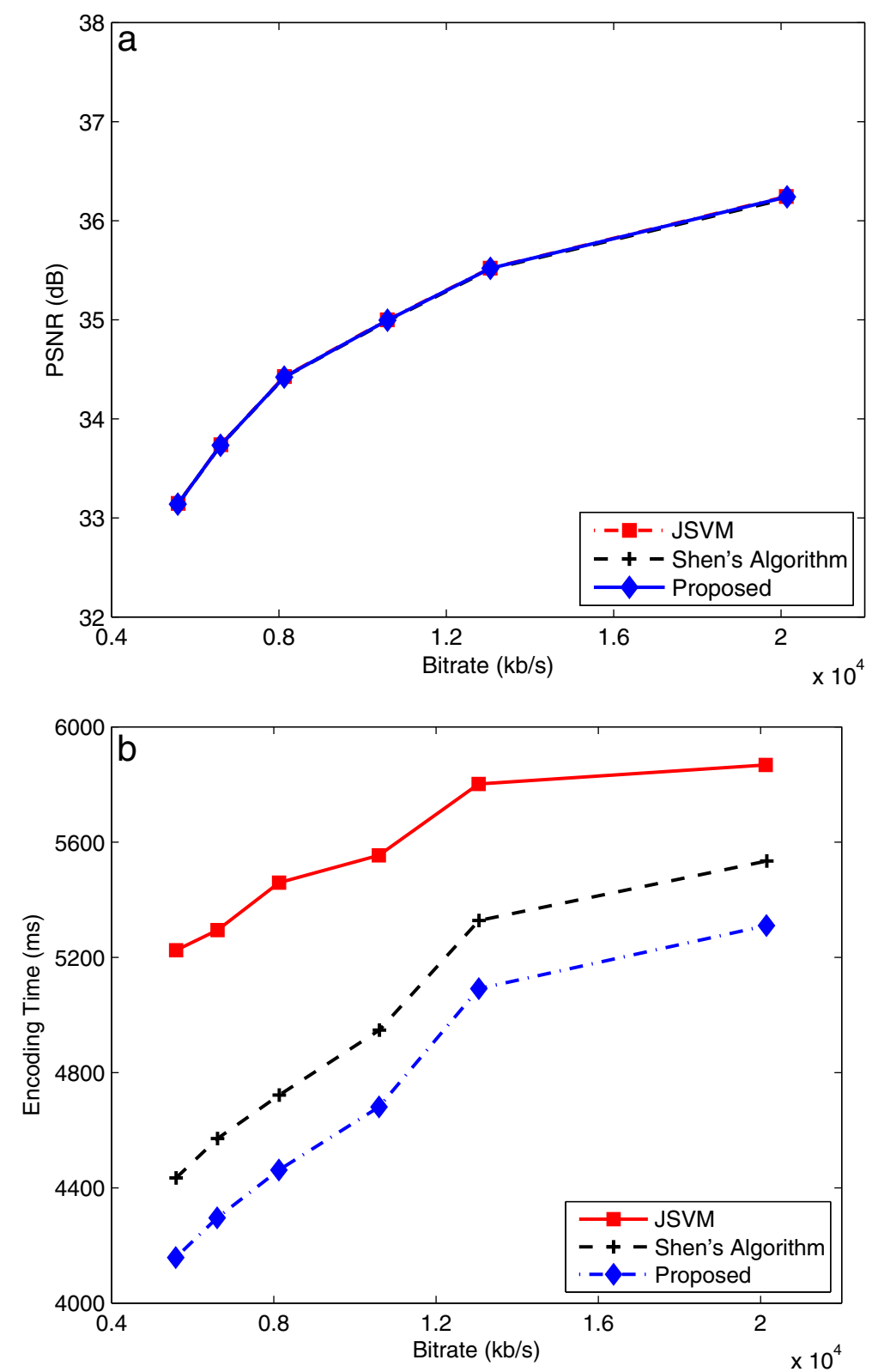

Figure 5 Comparison of RD performance (a) and encoding time per frame (b) for 'Shields' when DQP $=2$ 
Step 3) If CBP is non zero, goto to STEP 5; otherwise check BL_SKIP mode and goto to STEP 6.

Step 4) If Equations (2) and (3) are not satisfied for $\mathrm{Vs}=(1,1,0)$ and $\mathrm{Vs}=(1,0,1)$, respectively, goto STEP 5; otherwise, check BL_SKIP mode and goto STEP 6.

Step 5) Conduct the normal exhaustive mode decision for the rest of modes and determine the best mode.

Step 6) Go to STEP 1 to process the next MB.

During the encoding process, SKIP RD cost for each $\mathrm{MB}$ should be saved for the computation. Moreover, if
Vs $=(1,1,0)$ or $(1,0,1)$ and the left $M B$ is not available, the right neighbor of the top $M B$ would replace the left $M B$. On the other hand, if the right $M B$ is not available, the left neighbor of the left $\mathrm{MB}$ would be used instead.

\section{Experimental results}

The configurations for the simulation is listed in Table 4. Sequences with various types of resolutions and motions are used. Sequence 'Akiyo', 'Paris', and 'Silent' are of CIF format, 'Vidyo1', 'Shields' and 'Mobcal' belong to HD720p format. 'Vidyo1' is a test sequence used for the development of the ongoing standard High efficiency
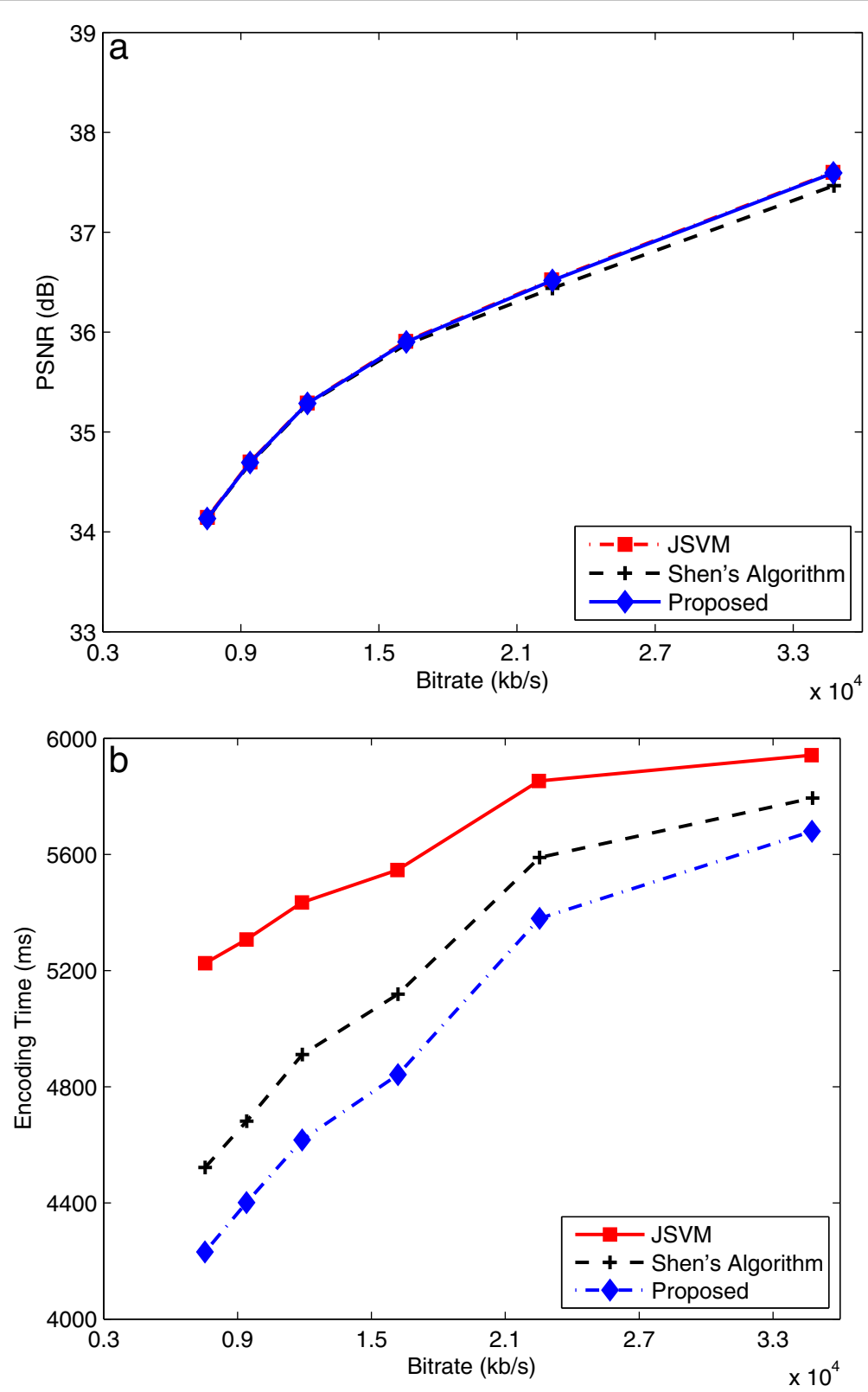

Figure 6 Comparison of RD performance (a) and encoding time per frame (b) for 'Shields' when DQP $=5$. 
video coding (HEVC)[19]. The QP differences are set to 2, 5 , and 10 and higher DQP means large quality difference between BL and EL.

JSVM 9.19 is used for the two layer CGS encoding. The simulation environment is a PC of $2.2 \mathrm{GHz} C P U$ and 8 GB memory. We use average bit rates increase (BDBR, $\%)$ and average peak-signal-to-noise ratio decrease (BDPSNR, $\mathrm{dB}$ ) to measure the average quality change [20]. We measure the total encoding time of $\mathrm{BL}$ and EL to reveal the speed of encoder. The average encoding time saving (ATS, $\%$ ) is used to measure the reduction on encoding complexity, which is the average of time savings (TS) for various
QPs. These parameters are important to compare the proposed fast approach with the reference software and the state-of-the-art fast algorithm. The results are obtained for various sequences.

Table 5 lists the results when comparing the proposed approach with JSVM. It demonstrates that in average, the proposed approach achieves $35.15 \%$ reduction of encoding time with $0.011 \mathrm{~dB}$ BDPSNR and 0.589\% BDBR loss. For slow motion sequences like "Akiyo", the reduction could be as high as $56.52 \%$.

Comparing with Shen's algorithm, the proposed approach achieves $9.98 \%$ time reduction with 0.045 BDPSNR
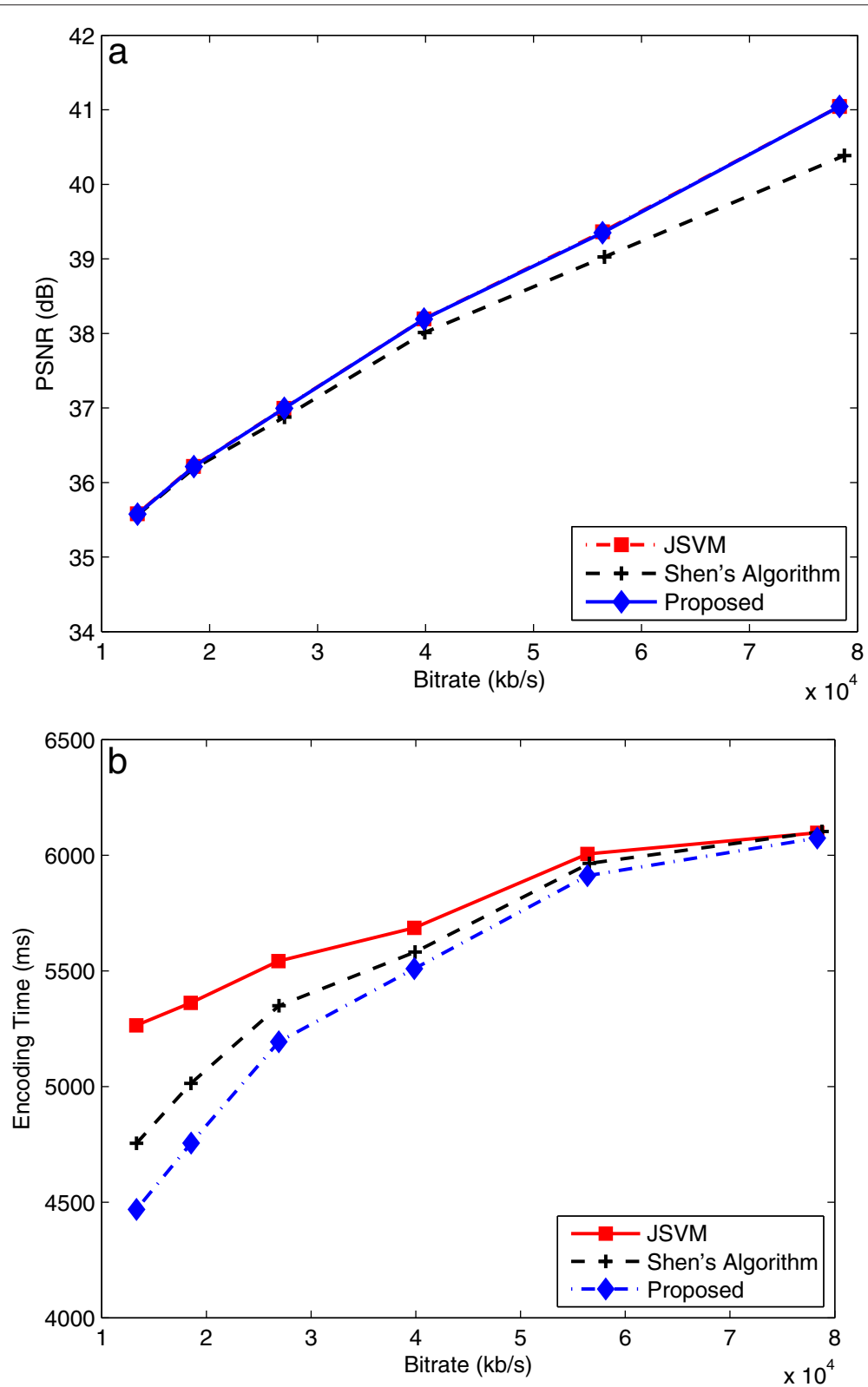

Figure 7 Comparison of RD performance (a) and encoding time per frame (b) for 'Shields' when DQP $=10$. 
and $0.879 \%$ BDRATE gain decrement on average (Table 6). Specifically, the maximum time reduction $15.74 \%$ is achieved for sequence "Akiyo" at DQP $=2$ with $0.036 \mathrm{~dB}$ BDPSNR and 0.4\% BDRATE gain. Overall, in half cases, the time reduction is more than $10 \%$ with negligible quality changes. Shen's algorithm makes a skip decision at EL only when all the EL neighboring MBs and the collocated MB at BL are skipped. This constraint excludes many candidates from early skip. Our proposed algorithm only requires the collocated $\mathrm{MB}$ and one of the EL neighboring MBs to be skipped, hence enlarged the candidate set. Combined this relaxation with other CBP and RD cost comparison, the proposed algorithm achieves better performance. Besides, the proposed algorithm sometimes achieves RD gains over Shen's algorithm as well as time reduction. We think it is because the algorithm sometimes checks BL_SKIP mode other than SKIP mode.

It is also interesting to see separate contributions of each part of the proposed algorithm. Because the RD cost comparison is interleaved with skip pattern detection, we only compare the algorithm with and without zero CBP detection and the results are listed in Table 7 for HD sequences. On average, the algorithm without and with zero CBP detection achieves 23.22 and $24.51 \%$ time reduction respectively with similar quality losses. Therefore, zero CBP detection provides about $1.29 \%$ time reduction.

Aside from the average performance metrics shown above, we choose one sequence from each resolution to show their RD performance and encoding time reduction for various bitrates. Figures 2, 3, and 4 show the RD performance curves for 'Paris' with CIF resolution. It can be seen that all three algorithms have similar RD curves. Encoding time per frame is displayed in Figures $2 \mathrm{~b}, 3 \mathrm{~b}$, and $4 \mathrm{~b}$ for each DQP. The proposed algorithm demonstrates much faster encoding speed than JSVM and Shen's algorithm under low and medium bitrates. It can be observed that the encoding time reduction tends to be smaller for higher bitrates. It is due to the fact that smaller number of MBs are skipped with higher bitrates. Similarly, 'Shields' is selected as the candidates of sequences with HD720p resolution. Figures 5, 6, and 7 show similar curves for 'Shields.' For sequence 'Shields', when DQP $=5$, 10, both Shen's and the proposed algorithm do not show much improvement under high bitrate scenarios. Further study is needed to improve encoding speed for the high bitrate case.

\section{Conclusion}

We propose a novel early skip algorithm in this article. By exploring extra skip patterns in the co-located MB at $\mathrm{BL}$ and spatial neighboring MBs at EL, further encoding speed improvement is achieved. The results demonstrate that, when combining with RD cost comparison and CBP checking, a larger portion of MBs in SKIP or BL_SKIP mode could be early detected with high precision. Compared with the reference software and the state-of-the-art algorithm, the proposed algorithm leads to greater encoding speed improvement and negligible quality losses. For further speed up, this approach could be used as the early skip detection module in other SVC FMD algorithms. In the future, we are interested in exploring other mechanisms to speed up SVC encoders such as fast motion estimation [21-24].

\section{Competing interests}

The authors declare that they have no competing interests.

Received: 14 July 2012 Accepted: 7 December 2012

Published: 4 January 2013

\section{References}

1. H Schwarz, D Marpe, T Wiegand, Overview of the Scalable Video Coding Extension of the H.264/AVC Standard. IEEE Trans. Circ. Syst. Video Technol. 17, 1103-1120 (2007)

2. A Eleftheriadis, R Civanlar, O Shapiro, Multipoint videoconferencing with scalable video coding. J. Zhejiang Univ. Sci. A. 7, 696-705 (2006)

3. M Wien, H Schwarz, T Oelbaum, Performance analysis of SVC. IEEE Trans. Circ. Syst. Video Technol. 17, 1194-1203 (2007)

4. J Lee, B Jeon, in IEEE International Conference on Multimedia and Expo(ICME), vol. 2. Fast mode decision for H.264, Taipei, Taiwan, 2004), pp. 1131-1134

5. I Choi, J Lee, B Jeon, Fast coding mode selection with rate-distortion optimization for MPEG-4 Part-10 AVC/H.264. IEEE Trans. Circ. Syst. Video Technol. 16, 1557-1561 (2006)

6. C Kim, H Shih, C Kuo, in IEEE International Conference on Image Processing (ICIP), vol. 2. Feature-based intra-prediction mode decision for H.264, Singapore, 2004), pp. 769-772

7. D Wu, F Pan, K Lim, S Wu, Z Li, X Lin, S Rahardja, C Ko, Fast intermode decision in H.264/AVC video coding. IEEE Trans. Circ. Syst. Video Technol. 15, 953-958 (2005)

8. C Kannangara, I Richardson, M Bystrom, J Solera, Y Zhao, A MacLennan, R Cooney, Low-complexity skip prediction for H.264 through Lagrangian cost estimation. IEEE Trans. Circ. Syst. Video Technol. 16, 202-208 (2006)

9. $\mathrm{H} \mathrm{Li}, \mathrm{Z} \mathrm{Li}, \mathrm{C}$ Wen, Fast mode decision algorithm for inter-frame coding in fully scalable video coding. IEEE Trans. Circ. Syst. Video Technol. 16, 889-895 (2006)

10. C Park, B Dan, H Choi, S Ko, A statistical approach for fast mode decision in scalable video coding. IEEE Trans. Circ. Syst. Video Technol. 19, 1915-1920 (2009)

11. S Jung, S Baek, C Park, S Ko, Fast mode decision using all-zero block detection for fidelity and spatial scalable video coding. IEEE Trans. Circ. Syst. Video Technol. 20, 201-206 (2010)

12. H Lin, W Peng, H Hang, Fast context-adaptive mode decision algorithm for scalable video coding with combined coarse-grain quality scalability (CGS) and temporal scalability. IEEE Trans. Circ. Syst. Video Technol. 20, 732-748 (2010)

13. B Lee, M Kim, A low complexity mode decision method for spatial scalability coding. IEEE Trans. Circ. Syst. Video Technol. 21, 88-95 (2011)

14. T Zhao, H Wang, S Kwong, CC Kuo, W Halang, Hierarchical B-picture mode decision in H.264/SVC. J. Visual Commun. Image Represent. 22, 627-633 (2011)

15. H Lin, H Hang, W Peng, Fast bi-directional prediction selection in H.264/MPEG-4 AVC temporal scalable video coding. IEEE Trans. Circ. Syst. Video Technol. 20, 3508-3523 (2011)

16. B Lee, M Kim, An efficient inter-prediction mode decision method for temporal scalability coding with hierarchical B-picture structure. IEEE Trans. Broadcast. 58(2), 285-290 (2012)

17. T Zhao, S Kwong, H Wang, CC Kuo, H.264/SVC mode decision based on optimal stopping theory. IEEE Trans. Image Process. 21(5), 2607-2618 (2012) 
18. L Shen, Y Sun, Z Liu, Z Zhang, Efficient SKIP mode detection for coarse grain quality scalable video coding. IEEE Signal Process. Lett. 17, 887-890 (2010)

19. G Sullivan, JR Ohm, in Proc. SPIE. Recent developments in standardization of high efficiency video coding (HEVC), (2010), p. 7798

20. G Bjontegaard, in VCEG-M33, vol. 6. ITU-T Q6/SG16. Calculation of average PSNR differences between RDcurves, (2001), p. 16

21. T Katayama, T Hamamoto, T Song, T Shimamoto, in International Technical Conference on Circuits/Systems, Computers and Communications. Improvement of motion estimation with modified search center and search range for H.264/SVC, Korea, 2009), pp. 401-404

22. T Katayama, T Hamamoto, T Song, T Shimamoto, Motion based lowcomplexity algorithm for spatial scalability of H.264/SVC, (HongKong, 2010), pp. 4213-4216

23. $\mathrm{S} \mathrm{Na}, \mathrm{C}$ Kyung, Activity-based motion estimation scheme for $\mathrm{H} .264$ scalable video coding. IEEE Trans. Circ. Syst. Video Technol. 20, 1475-1485 (2010)

24. L Shen, Z Zhang, Content-adaptive motion estimation algorithm for coarse-grain SVC. IEEE Trans. Image Process. 21(5), 2582-2591 (2012)

doi:10.1186/1687-5281-2013-2

Cite this article as: Zhang et al:: A novel early SKIP mode detection method for coarse grain quality in H.264/SVC. EURASIP Journal on Image and Video Processing 2013 2013:2.

\section{Submit your manuscript to a SpringerOpen ${ }^{\mathcal{O}}$ journal and benefit from:}

- Convenient online submission

- Rigorous peer review

- Immediate publication on acceptance

- Open access: articles freely available online

- High visibility within the field

- Retaining the copyright to your article

Submit your next manuscript at $\boldsymbol{\wedge}$ springeropen.com 